\begin{tabular}{ccc|} 
ISSN = 1980-993X - doi:10.4136/1980-993X \\
www.agro.unitau.br/ambi-agua \\
E-mail: ambi-agua@agro.unitau.br \\
Tel.: (12) 3625-4116
\end{tabular}

\title{
Economia de água na irrigação do coqueiro em função de áreas de maior concentração do sistema radicular e cobertura do solo (doi:10.4136/ambi-agua.65)
}

\author{
Ivandelson Siqueira Santos ${ }^{1}$; Carlos Alberto Vieira de Azevedo ${ }^{2}$; Hugo Orlando \\ Carvallo Guerra'; Frederico Antônio Loureiro Soares ${ }^{3}$; Vera Lucia Antunes de Lima²; \\ José Dantas Neto ${ }^{2}$ \\ ${ }^{1}$ Mestre em Eng. Agrícola, R. Antonio José Rodrigues, 81, Mirante, CEP 58104-320, Campina Grande, PB \\ E-mail: ivandelson@bol.com.br \\ 22DEAg/CTRN/UFCG. Av Aprígio Veloso, 882, Bodocongó, CEP 58109-970, Campina Grande, PB \\ Email: \{cazevedo, antunes, zedantas\}@deag.ufcg.edu.br; hugo_carvallo@hotmail.com \\ ${ }^{3}$ Instituto CENTEC. Av. Dr. Guarani, 313, Bairro Betânia, CEP 62040-730, Sobral, CE \\ E-mail: fredalsoares@hotmail.com
}

\section{RESUMO}

A produção brasileira de coco não tem sido suficiente para atender a demanda do mercado interno, o que tem obrigado a importar volumes expressivos de coco seco e semiindustrializado (IBGE, 2006). Como a região Nordeste destaca-se com a maior produção e é caracterizada por uma elevada evapotranspiração e baixa precipitação, faz-se necessário otimizar a utilização da água usada na irrigação do coqueiro. Na fase de desenvolvimento do coqueiro grande parte do sistema radicular se concentra lateralmente em até $1 \mathrm{~m}$ de raio, chegando na fase adulta a um raio de até $2 \mathrm{~m}$. O cálculo do volume de água aplicado na irrigação é normalmente calculado, considerando-se a área determinada pelo espaçamento da cultura e um coeficiente de cobertura de sua copa, o que faz com que grandes volumes de água sejam aplicados. Visando economizar água, esta pesquisa teve como objetivo calcular o volume de água de irrigação utilizando ás áreas de maior adensamento do sistema radicular. Para o primeiro ano de desenvolvimento da cultura do coqueiro, foram irrigadas, no campo, 4 bacias, delimitadas por anéis de zinco com os diâmetros de 0,7; 0,8; 0,9 e 1,0 m, consideradas de maior concentração do sistema radicular, e duas condições de cobertura de solo (coberto e $\mathrm{nu})$, mais uma testemunha $(4 \times 2+1)$, perfazendo nove tratamentos com três repetições, totalizando 27 parcelas. O cálculo do volume de água de irrigação na cultura do coqueiro, baseado nas áreas de maior concentração do sistema radicular, promoveu considerável economia de água com valores máximos e mínimos, respectivamente, de 93,95 e 87,62\%, na condição de solo sem cobertura e de 96,98 e 93,81\%, em solo com cobertura., em relação ao procedimento tradicionalmente utilizado. A cobertura da superfície do solo contribuiu para uma redução substancial do volume de água utilizado pelo coqueiro.

Palavras-chave: eficiência no uso da água; Cocos nucifera; área molhada.

\section{Coconut irrigation water saving as a function of areas of the concentration of the root system and soil cover}

\section{ABSTRACT}

The Brazilian production of coconut has not been sufficient to attend the internal market demand which has resulted in great importations of dry and semi industrialized coconut 
(IBGE, 2006). The northeast of Brazil has the greatest coconut production and is, at the same time, characterized for its high evapotranspiration demand and the lowest precipitation, therefore, it is necessary to optimize the irrigation water used. During the development phase of the coconut tree, most of the root system concentrates around $1 \mathrm{~m}$ of radius, and in the adult phase, in larger part, to a radius up to $2 \mathrm{~m}$. The traditional procedure of calculation of the water volume to be applied through irrigation, normally considers the area given by the crop planting spacing and a canopy cover coefficient. This results in great volumes of water applied unnecessarily. To save water, this study investigates the calculation of the volume of irrigation water considering the areas where larger concentration of the root system occurs. For the first year of development of the coconut tree, four areas of irrigation delimited by rings of zinc with diameters of $0.7 \mathrm{~m}, 0.8,0.9$ and $1.0 \mathrm{~m}$ were used to irrigate the portion with the larger concentration of the root system, and two conditions of soil covering (covered and bare soil), plus a control $(4 \times 2+1)$, resulting in nine treatments with three repetitions, totaling 27 plots. The procedure proposed in this research for the calculation of the water volume to be applied in the coconut crop, based on areas of larger concentration of the root system, promoted considerable economy of water, in relation to the procedure traditionally used. The isolation of the soil surface in order to avoid the evaporation contributed to a substantial reduction of the applied volume of water as well.

Keywords: irrigation; Cocos nucifera; wetted area.

\section{INTRODUÇÃO}

O coqueiro (Cocos nucifera L.) foi introduzido no Brasil em 1553, procedente da ilha de Cabo Verde, sendo no Nordeste onde a espécie encontrou melhor adaptação. O coqueiroanão, com aptidão para água ou coco verde, foi introduzido somente em 1925 (Ferreira et al., 1997). A região Nordeste é onde está concentrada a maior produção de coco do país. Em 2002, com uma produção de 1.928.236 milhões de frutos, essa região respondeu por $79 \%$ da produção nacional de coco (IBGE, 2006).

A produção brasileira, entretanto, não tem sido suficiente para atender à demanda do mercado interno, o que tem resultado, como conseqüência, em importações de volumes expressivos de coco seco e semi-industrializado (IBGE, 2006). Como a região Nordeste destaca-se com a maior produção e esta é caracterizada por ter maior evapotranspiração e menor precipitação, faz-se necessário aperfeiçoar os sistemas de irrigação e diminuir os grandes volumes de água hoje aplicados na agricultura.

A cultura do coco exige durante seu crescimento vegetativo e fase de produção grande quantidade de água de boa qualidade; sendo assim, dificilmente se encontrará água disponível em quantidades adequadas para atender à demanda evapotranspirativa em condições de cultivo em sequeiro. O regime pluvial ideal é aquele que apresenta precipitação média anual de $1500 \mathrm{~mm}$, com valores mensais nunca inferiores a $130 \mathrm{~mm}$. Um período de três meses, com menos de $50 \mathrm{~mm}$ de precipitação por mês, é essencialmente prejudicial ao coqueiro (Ferreira et al., 1997).

Estudos realizados por Miranda et al. (1999) em Paraípaba, CE, indicaram que, plantas jovens de coqueiro-anão irrigadas por micro aspersão, o consumo de água variou de 8 a $12 \mathrm{~L}$ por planta por dia nos primeiros 6 meses após o plantio (com até $10 \%$ da superfície do solo coberta pela cultura), de 12 a $28 \mathrm{~L}$ por planta por dia dos 7 aos 12 meses (10 a $16 \%$ de cobertura do solo), de 30 a 100 L por planta por dia dos 13 aos 24 meses de idade (16 a 36\% de cobertura do solo) e de 103 a 173 L por planta por dia dos 25 a 36 meses (36 a 64\% de 
cobertura do solo). A grande maioria dos estudos a respeito das necessidades hídricas do coqueiro tem sido realizada nas condições edafo-climáticas da Ásia, utilizando variedades gigantes. No entanto, no Brasil, a maior parte dos plantios irrigados de coqueiro utiliza a variedade anã, e assim os estudos encontram-se ainda em fase inicial. Dispor de dados sobre o sistema radicular do coqueiro é essencial para o dimensionamento e manejo da irrigação. A falta dessas informações faz com que o volume de água aplicado seja inadequado. Por outro lado o manejo da irrigação pode também interferir na formação do sistema radicular (Nogueira et al., 1997).

O uso de cobertura de plástico tem apresentado importantes resultados econômicos, uma vez que evitam capinas e se diminuem às perdas de água por evaporação, constituindo-se, assim, uma importante alternativa para economia de água na agricultura, principalmente, para as regiões semi-áridas, onde ocorrem baixos índices de precipitação e elevadas temperaturas Cermeño (1990) e Sganzerla (1991), constataram que melhores resultados foram conseguidos com filmes de polietileno que com materiais vegetais.

No Brasil, os primeiros registros da utilização de filmes de polietileno datam de 1967, no cultivo de morango (Goto, 1997). Nos últimos anos, o uso dos filmes tem-se expandido para o cultivo de diversas hortaliças, como pimentão, tomate, pepino, melão e abobrinha. Pinto (1997) trabalhando com diferentes tipos de cobertura de polietileno, com a cultura de alface, concluiu que em média, a evapotranspiração nos tratamentos com mulch de polietileno apresentou-se 25\% menor que nos tratamentos sem mulch.

Esta pesquisa teve como objetivo avaliar, sob condições de solo coberto com plástico e de solo nu, volumes de água, a ser aplicado na cultura do coqueiro-anão que consideram apenas a área de maior concentração do sistema radicular, visando a sua formação num determinado volume de solo delimitado por anéis de zinco.

\section{MATERIAL E MÉTODOS}

O experimento foi conduzido no município de Pocinhos, $\mathrm{PB}$, situado nas coordenadas $36^{\circ} 06^{\prime} 34^{\prime \prime}$ Oeste e $7^{\circ} 07^{\prime} 12^{\prime}$ ' Sul, com solos classificados como Neossolo textura média fase caatinga hiperxerófila relevo plano e suave ondulado e Litólico Eutrófico com fraca textura arenosa e/ou média fase pedregosa e rochosa caatinga hiperxerófila relevo suave ondulado substrato gnaisse e granito (EMBRAPA, 1999). O solo utilizado na presente pesquisa foi o Neossolo.

O delineamento experimental foi em blocos ao acaso, resultante das combinações fatoriais entre as quatro áreas delimitadas por anéis de zinco com diâmetros de 0,7;0,8; 0,9 e $1,0 \mathrm{~m}$, nas quais a água foi aplicada, e duas condições de cobertura de solo: coberto (CC) e solo nu (SC), mais uma testemunha $(4 \times 2+1)$, perfazendo nove tratamentos com três repetições, totalizando vinte e sete parcelas.

A testemunha foi uma bacia de irrigação com características, medidas e manejo idênticos às utilizadas pelos agricultores da região; uma bacia sem cobertura de solo, de 1,0 m de diâmetro irrigada diariamente com uma media de 43 litros de água.

Os anéis de zinco foram enterrados no solo $0,20 \mathrm{~m}$, deixando acima da superfície do solo uma altura de $0,35 \mathrm{~m}$. A área onde foi instalado o experimento foi dividida em 3 blocos, correspondendo às 3 repetições, cada uma com dimensões de 32 x $40 \mathrm{~m}$, possuindo 9 plantas, totalizando $1280 \mathrm{~m}^{2}$. Todos os blocos foram separados por uma bordadura de 9 plantas.

No meio da área do experimento enterrou-se uma tubulação com 12 pontos de distribuição de água, nos quais se conectava uma mangueira de plástico flexível de $50 \mathrm{~m}$ de comprimento fazendo a irrigação manualmente, usando como medida de volume de água um 
balde de plástico graduado em litros. A água de irrigação usada no experimento era proveniente de um poço tubular provido de catavento, com $55 \mathrm{~m}$ de profundidade e diâmetro de $150 \mathrm{~mm}$, que abastecia uma caixa de água com capacidade para $5 \mathrm{~m}^{3}$, situado a uma distância de $120 \mathrm{~m}$ da fonte de água. Inicialmente, o solo foi colocado a capacidade de campo, saturando para isso o solo e deixando drenar durante 24 horas.

Nos tratamentos com cobertura o solo foi coberto com uma lâmina de polietileno de dupla face (preta e branca), com a parte branca virada para cima para refletir a luz do sol, amenizando, assim, a alta temperatura no solo, e a parte de cor preta ficou virada para baixo para que o calor naquela região fosse absorvido.

O plantio foi realizado utilizando-se mudas com 6 meses de idade, apresentado raízes nuas e podadas no momento de serem plantadas, tratadas contra fungos e bactérias. Antes do plantio, as mudas permaneceram 10 dias na área experimental para adaptação climática e logo depois foi feito o plantio em covas espaçadas de $8 \mathrm{~m}$ entre linhas e $8 \mathrm{~m}$ entre fileiras com dimensões de $0,5 \times 0,5 \times 0,7 \mathrm{~m}$.

O volume de água de irrigação foi calculado conforme a equação [1] que substitui a equação de Howel e Hiller (1972) comumente usada.

$$
\mathrm{V}=\frac{3,14 \mathrm{R}^{2} \mathrm{~K}_{\mathrm{c}} \mathrm{K}_{\mathrm{p}} \mathrm{E}_{\mathrm{v}}}{\mathrm{E}_{\mathrm{a}}}
$$

em que:

$\mathrm{V}=$ Volume de água aplicado, $\mathrm{L}$ planta ${ }^{-1} \mathrm{dia}^{-1}$;

$\mathrm{R}$ = Raio do anel de zinco, em $\mathrm{m}$

Kc = Coeficiente de cultura;

$\mathrm{Kp}=$ Coeficiente do Tanque Classe A;

$\mathrm{Ev}$ = Evaporação do Tanque Classe A, mm;

Ea = Eficiência de aplicação de água.

Essa equação considera as áreas de maior concentração do sistema radicular do coqueiro.

A equação de Howell e Hiller que considera o espaçamento entre fileiras e plantas do coqueiro foi utilizada para calcular o volume de água aplicado na testemunha. Dada a dificuldade de se medir a evapotranspiração do solo coberto, este foi irrigado a cada 2 dias, assumindo-se assim que o consumo deste seria de $50 \%$ do solo sem cobertura. A irrigação nos tratamentos sem cobertura do solo foi diária é igual ao volume calculado pela equação [1].

$\mathrm{O}$ valor do Kc adotado para os 120 dias em que durou o experimento foi de 0,4, e o coeficiente de cobertura Cv de 20\%, conforme Nogueira et al. (1998). A evaporação Ev foi medida pelo uso de um Tanque Classe A instalado na área do experimento. A eficiência de aplicação de água foi considerada $100 \%$, uma vez que a água foi aplicada manualmente com um balde de plástico graduado em litros. O cálculo do volume de água a ser aplicado foi baseado em áreas de maior concentração do sistema radicular do coqueiro. Para tal, avaliaram-se 4 diferentes áreas delimitadas pelos anéis de zinco.

Os volumes de água aplicados e os parâmetros de crescimento do coqueiro (altura de plantas e diâmetro do caule) foram avaliados mediante análise de variância com teste $\mathrm{F}$ para os fatores diâmetro da área irrigada e condição de solo, segundo Ferreira (1991) e Santos et al. (1998). O fator quantitativo, área irrigada, foi submetido a análises de regressão. 


\section{RESULTADOS E DISCUSSÃO}

A Tabela 1 mostra um resumo das análises de variância e as médias da altura das plantas do coqueiro para os tratamentos estudados.

Tabela 1. Análise de variância para a altura das plantas do coqueiro para os diferentes diâmetros das áreas irrigadas e condições de cobertura do solo.

\begin{tabular}{|c|c|c|c|c|c|c|}
\hline \multirow{2}{*}{ Causas de Variação } & \multirow{2}{*}{ GL } & \multicolumn{5}{|c|}{ Quadrados médios } \\
\hline & & O DAT & 30 DAT & 60 DAT & 90 DAT & 120 DAT \\
\hline Diâmetro (D) & 3 & $897,01^{\mathrm{ns}}$ & $766,13^{\mathrm{ns}}$ & $738,79^{\mathrm{ns}}$ & $701,46^{\mathrm{ns}}$ & $711,79^{\mathrm{ns}}$ \\
\hline Condiç̧̃̃es de Solo (CS) & 1 & $260,04^{\mathrm{ns}}$ & $345,80^{\mathrm{ns}}$ & $227,55^{\mathrm{ns}}$ & $225,71^{\mathrm{ns}}$ & $247,04^{\mathrm{ns}}$ \\
\hline Interação D x CS & 3 & $510,32^{\mathrm{ns}}$ & $493,99^{\mathrm{ns}}$ & $446,02^{\mathrm{ns}}$ & $450,09^{\mathrm{ns}}$ & $458,82^{\mathrm{ns}}$ \\
\hline Testemunha x Fatorial & 1 & $159,47^{\mathrm{ns}}$ & $140,97^{\mathrm{ns}}$ & $223,06^{\text {ns }}$ & $230,23^{\mathrm{ns}}$ & $237,72^{\mathrm{ns}}$ \\
\hline Blocos & 2 & $612,71^{\mathrm{ns}}$ & $506,83^{\mathrm{ns}}$ & $417,29^{\mathrm{ns}}$ & $411,70^{\mathrm{ns}}$ & $407,86^{\mathrm{ns}}$ \\
\hline Resíduo & 16 & 383,12 & 432,30 & 405,38 & 393,42 & 390,67 \\
\hline $\mathrm{CV}(\%)$ & & 16,94 & 17,45 & 16,54 & 16,15 & 16,00 \\
\hline \multicolumn{2}{|c|}{ Diâmetro da Área Irrigada } & \multicolumn{5}{|c|}{ Altura da planta (cm) } \\
\hline & & \multicolumn{4}{|c|}{ Sem cobertura do solo } & \\
\hline $0,7 \mathrm{~m}$ & & 114,06 & 115,78 & 117,51 & 118,59 & 118,59 \\
\hline $0,8 \mathrm{~m}$ & & 133,40 & 135,49 & 139,48 & 140,42 & 140,42 \\
\hline $0,9 \mathrm{~m}$ & & 113,85 & 118,37 & 122,32 & 122,99 & 122,99 \\
\hline $1,0 \mathrm{~m}_{4}$ & & 87,61 & 91,58 & 96,04 & 97,81 & 97,81 \\
\hline \multirow[t]{2}{*}{ DMS } & & 3,53 & 3.40 & 3,27 & 3,23 & 3,23 \\
\hline & \multicolumn{5}{|c|}{ Com cobertura do solo } & \\
\hline $0,7 \mathrm{~m}$ & & 112,70 & 117,60 & 122,67 & 123,57 & 123,97 \\
\hline $0,8 \mathrm{~m}$ & & 120,33 & 122,50 & 123,67 & 124,50 & 125,33 \\
\hline $0,9 \mathrm{~m}$ & & 133,30 & 136,63 & 137,67 & 138,97 & 140,27 \\
\hline $1,0 \mathrm{~m}$ & & 112,43 & 118,20 & 119,17 & 120,53 & 121,70 \\
\hline DMS & & 41,65 & 54,07 & 53,58 & 51,83 & 52,83 \\
\hline
\end{tabular}

${ }^{\mathrm{ns}}$ não significativo. DAT - Dias após transplantio.

Observa-se que não houve diferença significativa na altura das plantas de coqueiro em nenhuma fase do crescimento do coqueiro para os diferentes diâmetros de área irrigada e tipos de cobertura do solo. Os coeficientes de variação ao redor de $16 \%$ permitem inferir uma boa exatidão do experimento. Assim, qualquer que seja o volume de água aplicado ou o tipo de cobertura do solo, a altura das plantas não foi afetada.

Assim como para a altura de planta, a análise de variância (Tabela 2) revelou que não houve diferença significativa tampouco para o diâmetro do caule das plantas de coqueiro para os diâmetros e tipos de condição de cobertura do solo. Considerando-se que um coeficiente de variação entre 12 e 15\% é classificado por Gomes (1970) como bom, isso indica que o delineamento estatístico utilizado exerceu bom controle sobre as variações do acaso. Assim, qualquer que seja o volume de água aplicada ou o tipo de cobertura do solo o diâmetro do caule das plantas não muda significativamente. 
SANTOS, I. S.; AZEVEDO, C. A. V.; GUERRA, H. O. C.; SOARES, F. A. L.; LIMA, V. L. A.; NETO, J. D. Economia de água na irrigação do coqueiro em função de áreas de maior concentração do sistema radicular e cobertura do solo. Ambi-Agua, Taubaté, v. 3, n. 3, p. 105-113, 2008. (doi:10.4136/ambi-agua.65)

Tabela 2. Resumo da análise de variância para o diâmetro do caule do coqueiro para os diferentes diâmetros da área irrigada e sob diferentes coberturas de solo.

\begin{tabular}{lcccccc}
\hline \multirow{2}{*}{ Causas de Variação } & \multirow{2}{*}{ GL } & \multicolumn{5}{c}{ Quadrados médios } \\
\cline { 3 - 7 } & 3 & $36,25^{\mathrm{ns}}$ & $6,81^{\mathrm{ns}}$ & $21,67^{\mathrm{ns}}$ & $9,81^{\mathrm{ns}}$ & $4,50^{\mathrm{ns}}$ \\
\hline Diâmetro (D) & 1 & $11,34^{\mathrm{ns}}$ & $0,01^{\mathrm{ns}}$ & $0,17^{\mathrm{ns}}$ & $3,38^{\mathrm{ns}}$ & $66,67^{\mathrm{ns}}$ \\
Condições de Solo (CS) & 3 & $25,12^{\mathrm{ns}}$ & $42,62^{\mathrm{ns}}$ & $41,25^{\mathrm{ns}}$ & $19,68^{\mathrm{ns}}$ & $57,00^{\mathrm{ns}}$ \\
Interação D x CS & 1 & $0,14^{\mathrm{ns}}$ & $5,57^{\mathrm{ns}}$ & $0,17^{\mathrm{ns}}$ & $3,63^{\mathrm{ns}}$ & $0,02^{\mathrm{ns}}$ \\
Testemunha x Fatorial & 2 & $17,29^{\mathrm{ns}}$ & $63,18^{\mathrm{ns}}$ & $8,07^{\mathrm{ns}}$ & $22,50^{\mathrm{ns}}$ & $25,59^{\mathrm{ns}}$ \\
Blocos & 16 & 12,83 & 10,59 & 17,42 & 21,38 & 26,30 \\
Resíduo & & 16,61 & 12,96 & 15,11 & 14,62 & 14,35 \\
\hline CV (\%) & & & & & & \\
\hline
\end{tabular}

\begin{tabular}{cccccc}
\hline Diâmetro da área irrigada & \multicolumn{5}{c}{ Diâmetro do caule (mm) } \\
\hline & \multicolumn{5}{c}{ Sem cobertura do solo } \\
\hline $0,7 \mathrm{~m}$ & 16,37 & 21,20 & 22,29 & 29,00 & 33,00 \\
$0,8 \mathrm{~m}$ & 22,17 & 28,00 & 29,48 & 32,67 & 41,33 \\
$0,9 \mathrm{~m}$ & 20,93 & 24,23 & 27,35 & 33,50 & 39,00 \\
$1,0 \mathrm{~m}$ & 23,93 & 27,67 & 30,47 & 32,33 & 36,33 \\
\hline & \multicolumn{5}{c}{ Com cobertura do solo } \\
\hline $0,7 \mathrm{~m}$ & 20,00 & 26,33 & 27,83 & 32,67 & 36,00 \\
$0,8 \mathrm{~m}$ & 20,67 & 23,00 & 25,00 & 27,50 & 30,33 \\
$0,9 \mathrm{~m}$ & 26,90 & 28,30 & 31,27 & 32,33 & 33,67 \\
$1,0 \mathrm{~m}$ & 21,33 & 23,50 & 26,83 & 32,00 & 36,17 \\
\hline
\end{tabular}

${ }^{\mathrm{ns}}$ não significativo. DAT - Dias após transplantio.

Na Tabela 3, são apresentados os volumes totais de água aplicados ao coqueiro durante os primeiros 120 dias após o transplantio.

Tabela 3. Volumes totais de água aplicados nos diferentes tratamentos e na testemunha durante os 120 dias após transplantio.

\begin{tabular}{ccc}
\hline TRATAMENTO & \multicolumn{1}{c}{ VOLUME DE ÁGUA APLICADO (L) } \\
\hline Diâmetro da área irrigada & Sem cobertura & Com cobertura \\
\hline $0,7 \mathrm{~m}$ & 310 & 154 \\
$0,8 \mathrm{~m}$ & 406 & 202 \\
$0,9 \mathrm{~m}$ & 513 & 256 \\
$1,0 \mathrm{~m}$ & 633 & 316 \\
Testemunha & 5120 & - \\
\hline
\end{tabular}

Obviamente, à medida que aumenta o diâmetro da área irrigada, aumenta o volume de água aplicada. A Tabela 3 mostra também que o solo sem cobertura recebeu o dobro de água que o solo com cobertura. Isso devido à freqüência de irrigação do solo sem cobertura que foi o dobro da freqüência do solo com cobertura.

A Tabela 4 apresenta os percentuais de economia no volume total de água aplicada promovidos pelos tratamentos para as diferentes condições de cobertura de solo e diâmetros da área irrigada em relação à testemunha. 
SANTOS, I. S.; AZEVEDO, C. A. V.; GUERRA, H. O. C.; SOARES, F. A. L.; LIMA, V. L. A.; NETO, J. D. Economia de água na irrigação do coqueiro em função de áreas de maior concentração do sistema radicular e cobertura do solo. Ambi-Agua, Taubaté, v. 3, n. 3, p. 105-113, 2008. (doi:10.4136/ambi-agua.65)

Tabela 4. Percentuais de economia no volume total de água aplicado.

\begin{tabular}{|c|c|c|c|c|c|c|}
\hline \multirow{2}{*}{$\begin{array}{l}\text { Diâmetro } \\
\text { molhado }\end{array}$} & \multirow{2}{*}{$\begin{array}{l}\text { Volume total } \\
\text { de água } \\
\text { aplicado (L) }\end{array}$} & \multicolumn{5}{|c|}{ Economia de Água (\%) } \\
\hline & & Trat. $^{1}$ & $\mathrm{SCC}^{2}$ & $\mathbf{D}_{1}{ }^{3}$ & $\mathbf{D}_{2}{ }^{4}$ & $\mathbf{D}_{3}{ }^{5}$ \\
\hline \multicolumn{7}{|c|}{ Sem cobertura do solo } \\
\hline $0,7 \mathrm{~m}$ & 310,01 & 93,95 & & & & \\
\hline $0,8 \mathrm{~m}$ & 405,70 & 92,08 & & 23,57 & & \\
\hline $0,9 \mathrm{~m}$ & 513,49 & 89,97 & & 39,63 & 20,990 & \\
\hline $1,0 \mathrm{~m}$ & 633,87 & 87,62 & & 51,09 & 36,00 & 18,99 \\
\hline \multicolumn{7}{|c|}{ Com cobertura do solo } \\
\hline $0,7 \mathrm{~m}$ & 154,60 & 96,98 & 50,13 & & & \\
\hline $0,8 \mathrm{~m}$ & 202,84 & 96,04 & 50,00 & 23,78 & & \\
\hline $0,9 \mathrm{~m}$ & 256,74 & 94,99 & 50,00 & 39,78 & 20,99 & \\
\hline $1,0 \mathrm{~m}$ & 316,93 & 93,81 & 50,00 & 51,22 & 36,00 & 18,99 \\
\hline
\end{tabular}

${ }^{1}$ Economia de água promovida pelo tratamento, em relação à testemunha.

${ }^{2}$ Economia de água com cobertura de solo. Economia de água usando: ${ }^{3} \mathrm{D}_{1},{ }^{4} \mathrm{D}_{2},{ }^{5} \mathrm{D}_{3}$, em relação aos diâmetros subseqüentes. SCC - solo com cobertura.

Na condição de solo sem cobertura, a máxima e a mínima economia de água foram, respectivamente, de 93,95\%, para o diâmetro de 0,7 m, e de $87,62 \%$, para o diâmetro de 1,0m. Para a condição de solo coberto, esses valores foram 96,98 e 93,81\%, respectivamente para os diâmetros de 0,7 e 1,0 m. Assim, conclui-se que o uso da menor área irrigada e, conseqüentemente, o menor volume de água produz a mesma altura de planta e diâmetro do caule que quando utilizada a maior área e com o maior volume. Isso se deve a que, embora o volume de água aplicado variasse, a lâmina de água se manteve sempre a mesma, pois foi calculada utilizando-se à evaporação do tanque Classe $\mathrm{A}$ em $\mathrm{mm}$, igual para todos os diâmetros.

Em termos relativos, os diâmetros molhados de 0,7; 0.8 e 0,9 m promoveram praticamente as mesmas economias de água nas condições de solo sem e com cobertura, que foram, respectivamente, em torno de 23 a 51\%, de 20 a 36\%, e de 18\%. Considerando-se que os diferentes volumes de água aplicada não produziram nenhuma variação significante nas variáveis de crescimento do coqueiro estudadas, recomenda-se a utilização do menor diâmetro de área irrigada a qual significará uma importante economia de água.

A irrigação a cada dois dias conduzida para o solo com cobertura originou uma economia de água de 50\%, com uma média diária para o volume de água aplicado, referente aos primeiros 120 dias após o transplantio, que variou de 1,29 a 2,64 L nos diferentes tratamentos; enquanto que para as condições de solo sem cobertura essa média variou de 2,58 a 5,28 L e para a testemunha foi de 42,67 L. Dessa forma, a cobertura da superfície do solo com o plástico de polietileno de dupla face efetivamente contribuiu, como esperado, para uma redução substancial no volume de água aplicado para atender às necessidades hídricas da cultura do coqueiro, tornando quase que todo o volume de água aplicado disponível à cultura. Esse fato mostra, então, a importância de se controlar a temperatura do solo em regiões semiáridas, evidenciando também a eficiência do filme de polietileno de duas faces (preta e branca) para essa finalidade, o qual cobriu a superfície do solo de forma que a parte branca ficou virada para cima para refletir a luz do sol, amenizando, assim, a alta temperatura no solo, e a parte de cor preta ficou virada para baixo para que o calor naquela região fosse absorvido. 


\section{CONCLUSÕES}

Os resultados obtidos permitem concluir:

1. O uso das áreas de maior concentração de raízes para o cálculo do volume de água a ser aplicado na cultura do coqueiro promoveu uma considerável economia de água, em relação ao procedimento tradicionalmente utilizado.

2. A cobertura da superfície do solo contribuiu para uma redução substancial do volume de água utilizado pelo coqueiro.

\section{REFERÊNCIAS}

AGRIANUAL. Anuário da agricultura brasileira. São Paulo: FNP, 2000. p.330-339.

EMPRESA BRASILEIRA DE PESQUISA AGROPECUÁRIA - EMBRAPA. Centro Nacional de Pesquisa de Solos. Sistema brasileiro de classificação de solos. Rio de Janeiro: Embrapa Solos, 1999. 412 p.

CERMEÑO, Z. S. Estufas: instalações e manejo. Lisboa: Litexa, 1990. 355p.

EMPRESA PERNAMBUCANA DE PESQUISA AGROPECUÁRIA - IPA. Recomendações de adubação para o Estado de Pernambuco. Recife: IPA, 1998. 198 p.

FERREIRA, P. V. Estatística experimental aplicada à agronomia. Maceió: UFAL/EDUFAL/FUNDEPES, 1991. 437 p.

FERREIRA, J. M. S.; WARWIK, D. R. N.; SIQUEIRA, L. A. A cultura do coqueiro no Brasil. Aracaju: Embrapa CPATC, 1997. 292 p.

GOMES, F. P. Curso de estatística experimental. Piracicaba: ESALQ-USP, 1970. 430p.

GOTO, R. Plasticultura nos trópicos: uma avaliação técnico-econômica. Horticultura brasileira, v. 15, p. 163-165, 1997.

HOWELL, T. A.; HILLER, E. A. Trickler Irrigation System Design. St. Joseph: ASAE, 1972. $221 \mathrm{p}$.

INSTITUTO BRASILEIRO DE GEOGRAFIA E ESTATÍSTICA - IBGE. SIDRA. Banco de Dados Agregados, Produção Agrícola Municipal. 2006. Disponível em: $<$ http://www.ibge.gov.br/bda/tabela/listabl.asp?c=1613\&z=t\&o= 11>. Acesso em: 22 agosto 2006.

MIRANDA, F. R.; OLIVEIRA, V. H.; MONTENEGRO, A. A. T. Desenvolvimento e precocidade de produção do coqueiro Anão (Cocos nucifera L.) sob diferentes regimes de irrigação. Agrotopica, v. 11, n. 2, p. 26-31, 1999.

NOGUEIRA, L. C; NOGUEIRA, L. R. Q.; MIRANDA, F. R. Irrigação do coqueiro. In: A cultura do coqueiro no Brasil. Aracaju: Embrapa CPATC, 1997. p. 159-187.

PINTO, J. P. Efeito do tipo de cobertura de polietileno preto na evapotranspiração e na produção da cultura de alface. Viçosa, 1997. 55p. Dissertação (M.S.) - Universidade Federal de Viçosa. 
SANTOS, I. S.; AZEVEDO, C. A. V.; GUERRA, H. O. C.; SOARES, F. A. L.; LIMA, V. L. A.; NETO, J. D. Economia de água na irrigação do coqueiro em função de áreas de maior concentração do sistema radicular e cobertura do solo. Ambi-Agua, Taubaté, v. 3, n. 3, p. 105-113, 2008. (doi:10.4136/ambi-agua.65)

SANTOS, J. W. dos; MOREIRA, J. de A. N.; BELTRÃO, N. E. M. Avaliação do emprego dos testes de comparação de médias na revista Pesquisa Agropecuária Brasileira de 1980 a 1994. Pesquisa Agropecuária Brasileira, v. 33, n. 3, p. 225-230, 1998.

SGANZERLA, E. Nova agricultura: a fascinante arte de cultivar com os plásticos. Porto Alegre: Plasticultura Gaúcha, 1991. 303 p.

THAMPAN, P. K. Success story in drip irrigation. Indian Coconut Journal, v. 21, n. 9, p. 810, 1991. 